\title{
On a Question of the Information Technology Construction Based on Self-learning Medicine Intelligent System
}

\author{
Anna Yankovskaya ${ }^{(1,2,3,4,5)}$, Ivan Gorbunov ${ }^{(2)}$, Ilya Hodashinsky ${ }^{(2)}$, Georgiy Chernogoryuk ${ }^{(5)}$ \\ ${ }^{(1)}$ Tomsk State University of Architecture and Building \\ ${ }^{(2)}$ Tomsk State University of Control Systems and Radioelectronics \\ ${ }^{(3)}$ National Research Tomsk Polytechnic University \\ ${ }^{(4)}$ National Research Tomsk State University \\ ${ }^{(5)}$ Siberian State Medical University \\ Tomsk, Russia \\ ayyankov@gmail.com, giv@keva.tusur.ru,hia@rambler.ru, chernogoryuk@yandex.ru
}

\begin{abstract}
A main construction approaches of an information technology-based self-learning intelligent system are described in the paper. The technology is designed to increase the accuracy of the differential diagnosis of bronchial asthma by using selflearning principle. Optimal combination of different approaches from mathematic, pattern recognition, probability theory, mathematical statistics, artificial intelligence and machine learning is the basis of the intelligent system.
\end{abstract}

Keywords-Information technology, intelligent system, selflearning, bronchial asthma, pattern recognition, decision-making, justification decision-making, cognitive tools, artificial intelligence, machine learning.

\section{INTRODUCTION}

A development of information technologies applied to various problem areas is one of the most important in the modern research. Necessity the development of information technology applied to solving medical tasks connected to a degradation of the health of the population.

Intelligent systems (IS) is a basis for many information technologies. World-renowned scientist D. A. Pospelov made enormous contribution to the IS development [1]. Features of the development of medical intelligent systems (IMS) are presented in publications $[2,3]$ at most detailed form.

Construction of self-learning IS is very relevant today. The main reason is using of self-learning IS allowing more accurate diagnostic decision-making, because a self-learning will increase the training sample representativeness.

We offer construction of self-learning intelligent medical systems (SIMS) on the basis of test methods of pattern recognition, as well as earlier constructed IS [4-8], because the test methods of pattern recognition require a much smaller size of training sample in compare with statistical methods [9].

SIMS constructracted with usage fuzzy logic increase the accuracy of decision-making when variables (symptoms) (symptoms) defined as fuzzy sets and it is good way for reflection medical data and knowledge specificity. Let us note that fuzzy set theory as part of soft computing was suggested by Lotfi Zadeh in 1965 and developed in paper [10].

Using different approaches, methods, techniques, kind of logic inference in construction SIMS leads to synergy effect and provides to the reasonable of hybrid SIMS (HYSIMS) construction.

A justification of decision-making as part intelligent systems have a very important significance. Usage cognitive tools allowing justify the decisions in static and dynamic SIMS to predict and simulate of the disease progress.

In the paper is described a problem background; mathematical basics of SIMS and HYSIMS; brief information about the construction and applications HYSIMS, as well as directions for further research.

\section{PROBLEM BACKGROUND}

Intelligent medical systems based on the different approaches with using of statistical methods [11], Bayesian networks [12], logical-combinatorial methods [13], logicalprobabilistic and logical-combinatorial-probabilistic methods [13], test methods of the pattern recognition [9, 13], fuzzy and threshold logics [14], genetic algorithms [14, 15], neural networks [14], etc. is used in science, education and medical practice for a long time.

The overwhelming majority of researches about models creation and software tools development in the field of the medicine is aimed at understanding of the disease and its diagnosis [11, 12, 16-18]. A smaller part of the carried out mathematical and computer researches with aims to methods development and forecasting tools [19, 20], and very small part carried out to complex rehabilitation choose [21, 22]. Figure 1 shows the distribution of the number of publications over the years, found in SCOPUS for search queries: «ComputerAssisted System for Medical Diagnosis», «Computer-Assisted

Russian Foundation for Basic Research, project №16-07-00859, 16-07-00034, 16-37-60051. 
System for Medical Prognosis», and «Computer-Assisted System for Medical Rehabilitation».

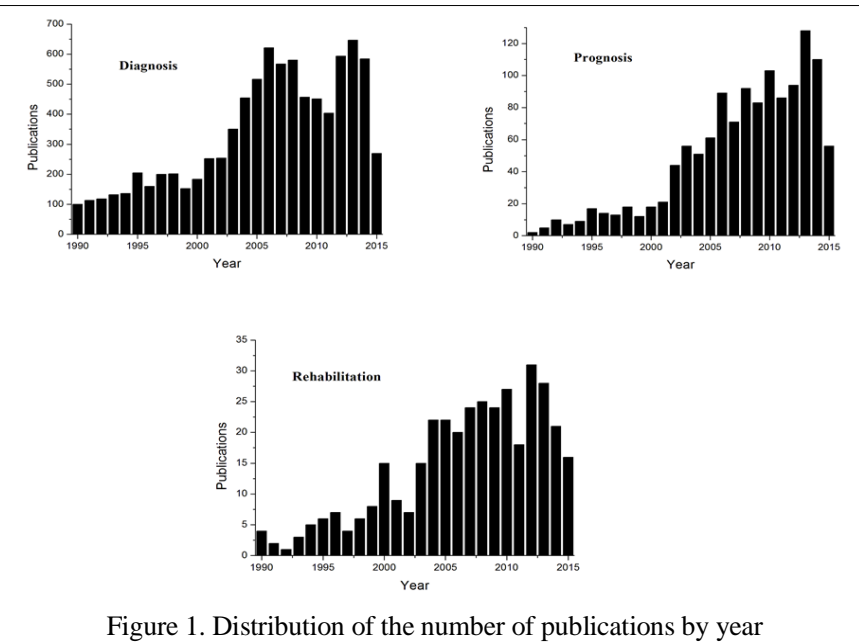

The publication about construction of the expert and intelligent decision-making system similar to our research is noted follow.

Recommendation system is described in the publication [22] which developed in the military department of Germany for diagnosis in the recruitment conscripts into the army; FEARDEX system is described in the publication [23] developed in the US for phobia diagnose; IS neural networkbased decision support system for pre-diagnosis of psychiatric disorders is described in the publication [24]; IS Intelligent decision support system for depression diagnosis based on neuro-fuzzy-CBR hybrid is described in the publication [25]; IS Beck depression inventory test assessment using fuzzy inference system is described in the publication [26]. Very significant results obtained by domestic scientists and presented in publications B. A. Kobrinskii, B. A. Kobrinskii [27], L. V. Kan, Y. M. Kuznetcova, N. V. Chudova [28].

To improve the predictive ability of the models of breast cancer are proposed to use a hybrid approach to classification in the paper [29]. An approach based on neuro-fuzzy ANFIS type system proposed to predict heart disease is described in the paper [30] with using a genetic algorithm.

The authors of review paper [31] note that the work on the application of machine learning methods for solving problems in the cancer diagnosis is ten times more than the works on this disease forecast. The authors make conclusion that usage of the machine learning for classifiers in medical practice will be much wider and more affordable.

Let us note the methods and inference systems similar to our research. Our research in the field of IS is conducted not from scratch. The more than 20 IS in medical, biology, sociology fields were developed under the leadership of A. E. Yankovskaya in the TSUAB (1) Intelligent Systems Laboratory. These IS are described in publications [4-8, 13, 32-34]. In additional we note our research at construction IMS with usage fuzzy classifiers is give in papers [15, 35-36].
Let us mention the main papers about the construction of self-learning intelligent systems similar to our work, but based on another approaches. The basic concept of self-learning evolving fuzzy systems was determined in papers of the following authors: P. Angelov, D. Filev, N. Kasabov, E.D. Lughofer, R. Yager in the early 2000s.

Most of evolving fuzzy systems are systems based on rules, in which the adaptation of knowledge base and optimize direction of antecedent and consequent parts of the rules takes place depending on the flow of incoming data. The adaptation of knowledge base is based on recursive clustering algorithms in most cases [37-39] today. Consequent parameters are updated with usage of the recursive least square method [40] in all known evolving fuzzy systems based on rules.

Two main directions of research can be identified in the field of evolving fuzzy systems: fuzzy self-learning systems based on rules [41] and self-learning neuro-fuzzy networks [42].

Cognitive tools used to construction the SIMS are given in the end of this section.

The main function of $n$-simplex is a representation of a disposition of object under study among other objects of a learning sample and the representation same distance between all objects under study. Additionally, n-simplex has other useful functions for a decision-making person. One of these functions is a representation of some numerical values, for example, an admissible error of recognition preassigned by the user [43].

Visualization of data and knowledge, as well as decisionmaking and decision justification on the basis of a 3-simplex is based on the theorem of conservation of the sum of the distances from the point to the edges of the n-simplex and the relationship between these distances [44-46]. Detailed description of visualization of objects with usage 3-simplex is presented in the paper [47].

\section{MATHEMATICAL BASIS OF THE CONSTRUCTION SIMS AND HYSIMS}

The basis of the most our IS is a matrix model representation of data and knowledge give in the paper [13]. The matrix model includes integer description matrix (Q) and distinguishing matrices $(\mathrm{R})$.

Rows of the matrix $\mathrm{Q}$ are associated with training objects, while columns - with characteristic features described each object. In addition, the element $q_{i j}$ of the matrix Q could has an undefined value (-) or has an interval of values ("dash").

Rows of the matrix $\mathrm{R}$ are associated with rows of the matrix $Q$ and columns - with classification features that specify levels of distinguish (classification mechanisms). Classification features take integer values and are interpreted as the numbers of classes to which objects belong.

The objects that have the same combination of classification feature values corresponding to a certain solution are assumed to belong to the same pattern. Thus, the number of not repeating rows of the matrix $R$ equal the number of patterns, and each subset of rows of the matrix $Q$ to which identical 
rows of the matrix $\mathrm{R}$ are associated with describe a certain pattern $[13,48]$.

Each pattern is assigned a number. The matrix R', which consists of one column, is used to indicate the patterns to which objects belong.

This model allows one to represent not only data but also expert knowledge, since one row of the matrix Q can define (in the interval form using the "dash" value) the subset of objects that have the same solution given by a corresponding row of the matrix $\mathrm{R}$.

A diagnostic test (DT) $[9,13,48,49]$ is a set of features that distinguish any pairs of objects that belong to different patterns. A diagnostic test is called non-redundant (dead end [9]) if it involves a non-redundant number of features. An unconditional non-redundant diagnostic test is characterized by the simultaneous presentation of all included features of an object under study for decision-making.

By regularities we mean the features that are constant (taking the same value for all patterns), stable (constant only within a pattern), non-informative (not distinguishing a single pair of objects), alternative (in terms of inclusion into the DT), dependent (in terms of inclusion of subsets of distinguishable pairs of objects), irrelevant (not included into a single nonredundant DT), obligatory (included into all non-redundant DTs), and pseudo obligatory (not obligatory but included into all UNDTs that are involved in decision making), fault tolerant (features that are tolerant to measurement errors), signal feature (signal feature first kind and signal feature second kind) as well as all minimum and all (or several for a large feature space) non-redundant distinguishing subsets of features that are essentially minimum DTs and non-redundant DTs, respectively. Weight coefficients [9] of characteristic features, as well as their information weights, are also called regularities $[9,13$, 48].

Unfortunately, the paper size limitations do not allow to mention the solid mathematical apparatus to identify different kinds of regularities, which include construction a fault-tolerant unconditional non-redundant diagnostic tests (FT UNDT) [13]; FT mixed diagnostic tests (FT MDT) [13] where MDT is a compromise between unconditional and conditional components; FT optimal subsets of signal characteristics (FT MSSC) [50] of the first and second kind; as well as way of the choice of optimal subsets (OS) FT UNDT; FT MDT [50]. Since the construction of the above-mentioned FT UNDT, FT MDT are reduced to the solution of NP-complete problems. On the set of OS FT UNDT and OS FT MDT are constructed decision rules, which used for final decision-making with usage of a voting procedure. The voting procedure is performed taking into account the weight coefficients of decision rules for each of the FT UNDT and FT MDT, as well as the weight of expert, which involved in construction distinguishing matrices (each expert construct one distinguishing matrix).

Decision-making on the object under study (examinee) is based on the decision-making rules. Current for this moment, description and distinguish matrices is supplemented with description of the object under study and result of the decision- making about it only in case the decision is made with an accuracy not exceeding the preassigned. This leads to increase the size of the training sample and will increase the accuracy of the decision-making for new object under study. That is proposed approach to self-learning.

Justification of the decision-making is based on our naturalistic [7, 13] and invariant to problem fields [13, 43, 47] of cognitive tools. Process of self-learning can be represented with using our cognitive tools.

Let us introduce the following notation to describe the principles of work Pittsburgh fuzzy classifier used as part as HYSIMS[51]: $\mathrm{x}=\left(x_{1}, x_{2}, x_{3}, \ldots, x_{n}\right)$ is vector of characteristic features; $A_{k i}$ - the membership function which characterizes $k$ feature in $i$-rule $(i \in\{1,2, \ldots, u\}), u$ is the number of rules; $\mathrm{m}$ is the number of classes; $\underline{c}_{i}$ is identifier of $j$-class, $j \in\{1,2, \ldots$, $m\} ; w$ is weigh of rule; $C F_{j i}$ is rule weighing coefficient or degree of belief of $j i$-rule, $C F_{j i}$ is defined at the interval $[0,1]$. rules:

Pittsburgh fuzzy classifier work based on following type of

$$
\begin{aligned}
& R_{j i}: \text { IF } x_{1}=A_{1 i} \text { AND } x_{2}=A_{2 i} \text { AND } x_{3}=A_{3 i} \text { AND ... AND } x_{n}=A_{n i} \\
& \text { THEN class }=c_{j}, w=C F_{j i} .
\end{aligned}
$$

The fuzzy classification is described by the function, which refers the classified object to $j$-th class with the definite grade of membership being calculated in the following way:

$$
\beta_{j}(\mathbf{x})=\sum_{R_{j i}}\left[\prod_{k=1}^{n}\left(A_{k i}\left(x_{k}\right)\right) \cdot C F_{j i}\right], j \in\{1,2, \ldots, m\} .
$$

The classifier output is the class being defined in the following way:

$$
\text { class }=c_{j^{*}} \text {, if } j^{*}=\arg \max _{1 \leq j \leq m}\left(\beta_{j}\right)
$$

Estimate of Pittsburgh fuzzy classifier confidence in its decision is calculated by the following formula:

$$
\mathrm{d}=\frac{\sum_{i=1}^{u} C F_{j i}}{u}
$$

Construction SIMS based on self-learned Pittsburgh fuzzy classifier named PFC was based on usage recursive mountain clustering method suggested by P. Angelov, D. Filev [52] and recurrent adaptive fuzzy clustering method of the Gustafson and Kessel [53].

\section{BRIEF INFORMATION ABOUT THE CONSTRUCTION AND APPLICATIONS HYSIMS}

Set of previously realized IMS and hybrid IMS [4-8, 32-34, 55] as component providing self-learning on the base abovementioned approaches is basis for construction SIMS and HYSIMS.

SIMS is constructed on the basis of intelligent instrumental software (tool) IMSLOG [54]. 
HYSIMS includes SIMS and PFC. The decision-making in the HYSIMS is carry out on the base of voting procedure taking into account the degree of reliability in accepted decisions by each of IS and confident levels of each decision of these IS.

HYSIMS is applicable for differential diagnosis in different kind of diseases. HYSIMS have been applying for differential diagnosis of asthma in the first time. Unfortunately, the paper size limitation does not allow describing more the representation of results of the HYSIMS with cognitive tools which are component of the HYSIMS.

Experts in the field bronchus asthma introduced the following naturalistic way of knowledge representation about the disease. Naturalistic way of cognitive knowledge representation follows when familiar graphic images of real objects are used by cognitive tools.

Knowledge is structured as a tree where ending tops are cross-sections (cuts) of the real world objects (bronchus) that characterize object's condition. Cross-section is colored with different colors and colored part is equal to the scale of the pathological process (spasm, edema of mucous, hypersecretion).

Perception of the bronchus condition comes out of the expert's individual experience and her knowledge of the pathological process's origin [55].

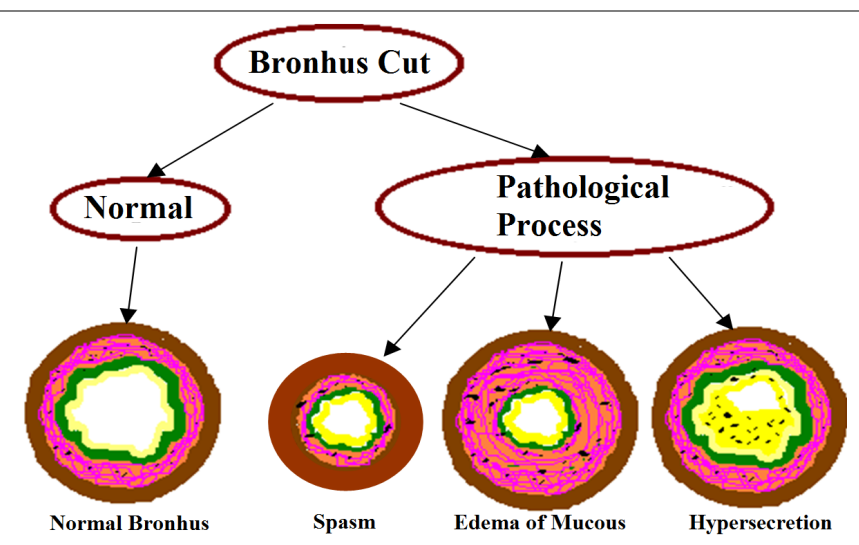

Figure 2. Naturalistic way: simple tree data structure of pathological processes in bronchus (bronchial asthma)

Figure 2 shows one graphic example relevant for differential diagnosis of asthma.

Since this figure displays the objects related to the four patterns, both the objects belonging to the corresponding pattern and the patterns are painted in different colors for better perception, as shown in Fig. 3:

Another way for represent descriptions manifestation degree of bronchial asthma different people under study are visualized on the base of the 3-simplex, as shown in Fig. 3 .

1) Pattern 1 (normal), corresponded to the lower edge is painted in red.

2) Pattern 2 (spasm), corresponded to the near left edge is painted in orange.

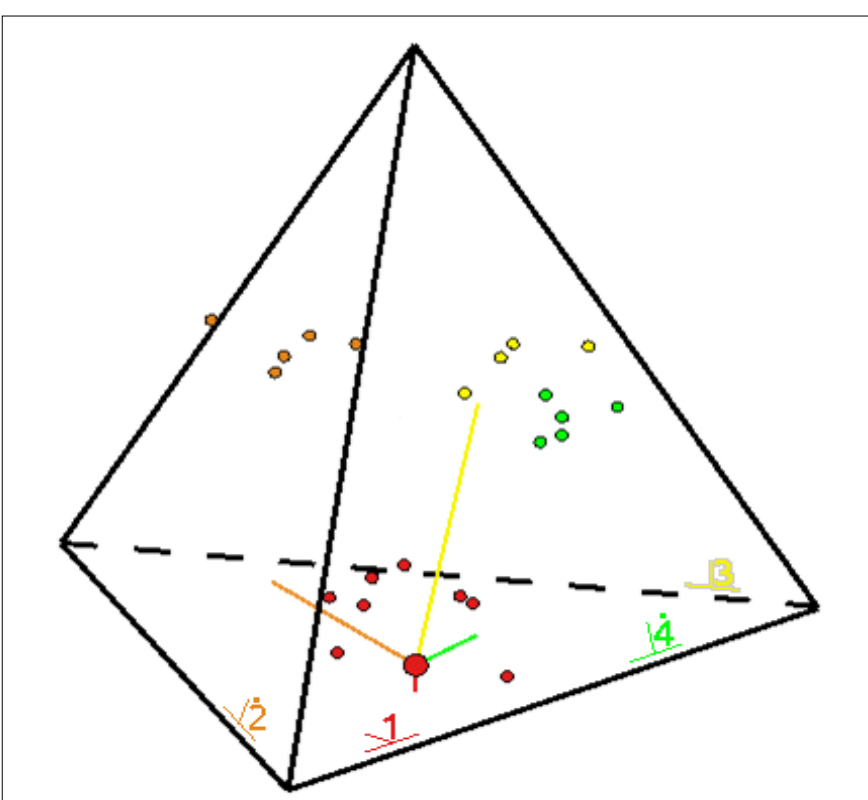

Figure 3. Non-naturalistic way: 3-simplex of manifestation degree of bronchial asthma

3) Pattern 3 (edema of mucous), corresponded to the distant edge is painted in yellow.

4) Pattern 4 (hypersecretion), corresponded to the near right edge is painted in green.

The same colors are used to display the distance from the object under study to the corresponding edge of the 3-simpex.

Points over the pattern numbers 2, 4 indicate that these 3simplex edges are visible.

The cognitive tool "2-simplex prism" is used for representation of different kind of dynamic processes in the HYSIMS.

The cognitive tool "2-simplex prism" (Figure 4) is based on 2-simplex and represents the right triangular prism which contains in basics and cuttings 2-simplexes which are corresponded fixed time moments.

Distance from the basis of the prism to $i$-th 2-simplex $h_{i}$ ' corresponds to the fixation moment of object under study features and it is calculated based on the following formula:

$$
h_{i}^{\prime}=H^{\prime} \cdot \frac{T_{i}-T_{\min }}{T_{\max }-T_{\min }}
$$

where $H^{\prime}$ - length of 2-simplex prism preassigned by a user and corresponded to the investigation duration, $T_{i}$ - timestamp of features fixation of object under study for $i$-th examination, $T_{\min }$ - timestamp of features fixation of object under study for the 1-st examination, $T_{\max }$ - timestamp of features fixation of object under investigation for the last examination. 


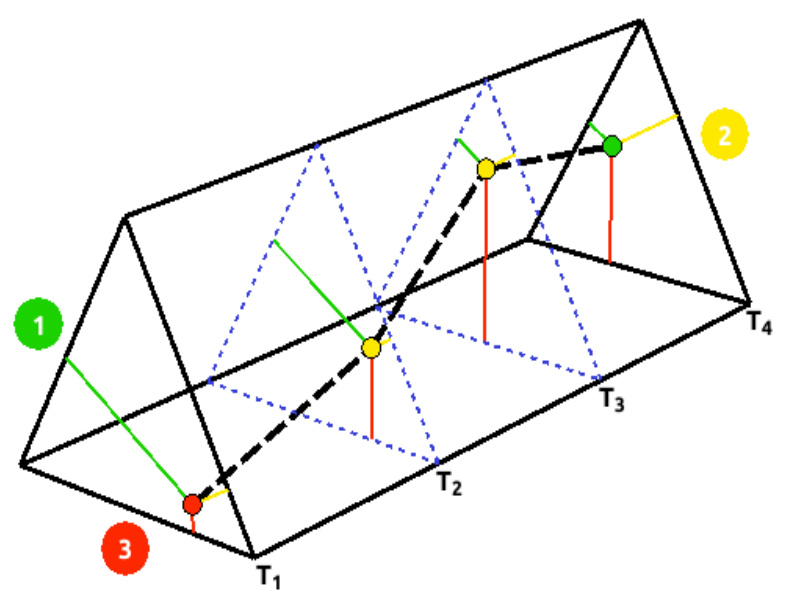

Figure 4.Example of 2-simplex prism usage for visualization of dynamical processes

Because 2-simplex prism is based on the 2-simplex description of all 2-simplex objects is also right for 2-simplex prism.

2-simplex prism allows representing visually as well the dynamic processes not only for medicine and for treatment process modeling/

Example of 2-simplex prism in medical application is given in the paper [44].

\section{$\mathrm{V} \quad$ CONCLUSION}

Basis of the construction self-learning intelligent medicine systems and hybrid self-learning intelligent medicine systems are given.

In the paper is detailed described an intelligent medical systems.

Mathematical apparatus of the construction SIMS and HYSIMS is given briefly and 2 approaches for self-learning as well as approaches for SIMS construction are proposed.

Optimal combination of different approaches from mathematic, pattern recognition, test methods of pattern recognition, probability theory, mathematical statistics, fuzzy and threshold logics, artificial intelligence and machine learning is the basis of HYSIMS. Effectiveness proposed combination approaches is beyond a question.

Using different approaches, methods, techniques, kind of logic inference in construction HYSIMS leads to synergy effect.

HYSIMS is constructed with usage of intelligent instrumental software (tool) IMSLOG.

Cognitive tools are used for a justification of decisionmaking in the SIMS, HYSIMS, also significance of cognitive tools are shown.

Usage cognitive tools allowing justify the decisions in static and dynamic SIMS to predict and simulate of the disease progress.

Proposed cognitive tools are based on 2 approaches: naturalistic and invariant to problem fields that important for application not only for medicine, but also for modeling one or another process which is necessary for a big amount of problems and cross-disciplinary areas: medicine, economy, genetics, building, radioelectronics, sociology, education, psychology, geology, design, ecology, geo-ecology, eco-biomedicine etc.

For justification of decision-making at dynamic processes proposed to use 2-simplex prism in SIMS and HYSIMS.

\section{Acknowledgment}

The work is supported by Russian Foundation for Basic Research, project №16-07-00859, 16-07-00034, 16-37-60051.

\section{References}

1 D. A. Pospelov, Artificial Intelligence, Vol. 2: Models and Methods, Radio i Szyaz, 1990, p. 304 (in Russian).

2 M. Elyanov catalog "Medical Information Technology 2015", URL: http://www.armit.ru/ (in Russian).

3 B. A. Kobrinskii, Features of the medical intellectual systems // Information-measuring and control systems, 2013, pp. 58-64 (in Russian).

4 A. E. Yankovskaya, R. V. Ametov, E. A. Muratova, G. E. Chernogoryuk, I. A. Mandel, Information technology for solving of problems connected on expert conclusion and construction of medical intelligent system on basis of this technology, Proceedings Of The Second IASTED International Multi-Conference Automation, Control, And Information Technology/Automation, Control, And Applications (ACIT-ACA). June 20-24, 2005, Novosibirsk, Russia, pp. 187-192.

5 A. E. Yankovskaya, G. E. Chernogoryuk, E. A. Muratova, Intelligent Test Recognizing Biomedical System, The 6th German-Russian Workshop "Pattern Recognition and Image Understanding" OGRW-62003, Workshop proceedings, Novosibirsk, Russian Federation, 2003, pp. 248-251.

6 A. E. Yankovskaya, S. V. Kitler and N. M. Krivdyuk, Knowledge and Data Acquisition and Processing in Solving Biomedical Problems, Pattern Recognition and Image Analysis, 2015, Vol. 25, No. 3, pp. 378384.

7 A. E. Yankovskaya, A. I. Gedike, N. N. Il'inskikh, G. E. Chernogoruk The Principles of the Development of an Intelligent Test Biomedical System, Pattern Recognition and Image Analysis, 2001, 11(2), pp. 484487.

8 A. I. Gedike, A. E. Yankovskaya Intelligent Ecology-Medical System //Problemy Upravleniya I Informatiki (Avtomatika), 1995, 5, pp. 224228.

9 Yu. I. Zhuravlev, I. B. Gurevitch, "Pattern recognition and image analysis," in Artificial Intelligence in 3 Books, Book 2: Models and Methods: Reference Book, Ed. by D. A. Pospelov (Radio and Comm., Moscow, 1990), pp.149-191 (in Russian).

10 L. Zadeh, Fuzzy logic and approximate reasoning. Synthese, 30, 1975, 407-428.

11 A. Belle, M, A. Kon, K. Najarian, Biomedical informatics for computeraided decision support systems: A survey. The Scientific World Journal, Article ID 769639, 2013, pp. 1-8. 
12 S. Montani, How to use contextual knowledge in medical case-based reasoning systems: A survey on very recent trends, Artificial Intelligence in Medicine, 51(2), 2011, pp. 125- 131.

13 A. E. Yankovskaya, Logical Tests and Means of Cognitive Graphics (LAP LAMBERT Acad. Publ., 2011), 2011, (in Russian).

14 L.A. Zinchenko, L.G. Komartsova, V.V. Kurejchik, V.M. Kurejchik, V.G Redko, S.N. Sorokin, Yu.R. Tsoy, A.E. Yankovskaya, N.G. Yarushkina "Bionic information systems and their practical application" Moscow: FIZMATLIT, 2011, pр. 288 стр. (ISBN: 978-5-9221-1302-1)

15 A. A. Zajcev, I. A. Hodashinsky, P. A. Dudin, D. S. Sinkov, I. V. Gorbunov, Methods of computational intelligence in predicting the effectiveness of non-drug treatment, Proceedings of the V International Scientific Conference "System Analysis in Medicine" (SAM 2011) (Blagoveshchensk, 2011). pp. 25-28 (In Russian).

16 I. Kim, J. Watada, Towards a new medical decision support system with bio-inspired interpretive structural modelling, New Advances in Intelligent Decision Technologies. Series Studies in Computational Intelligence, 199, 2009, pp. 459-466.

17 C. A. Pena-Reyes and M. Sipper, Evolutionary computation in medicine: an overview, Artificial Intelligence in Medicine, 19(1),2000, pp.1-23.

18 R.-J. Kuo, M.-H. Huang, W.-C. Cheng, C.-C. Lin, Y.-H. Wu, Application of a two-stage fuzzy neural network to a prostate cancer prognosis system, Artificial Intelligence in Medicine, 63(2), 2015, pp. 119-133

19 L. Minne, T. Toma, E. de Jonge, A. Abu-Hanna, Assessing and combining repeated prognosis of physicians and temporal models in the intensive care, Artificial Intelligence in Medicine, 57(2), 2013, pp. 111117.

20 R. van der Meer, Recent developments in computer assisted rehabilitation environments. Military Medical Research, 1, 2014, pp. 1-7.

21 L. Subirats, L. Ceccaroni, C. Gomez-Perez, R. Caballero, R. LopezBlazquez, F. Miralles, On semantic, rule-based reasoning in the management of functional rehabilitation processes, Management Intelligent Systems, Series Advances in Intelligent Systems and Computing, 220, 2013, pp. 51-58.

22 W. A. Sands, M. F. Wiskoff, Expert System Planning: German Federal Armed Forces Psychological Service, 1996, URL: http://www.ijoa.org/imta96/paper36.html

23 J. M. Amosig, E. J. Escara, R. Martinez, E. Paculanang, Feardex: Fear Diagnostic Expert System, 2008, URL: http://www.shvoong.com

24 Y. Bouaiachi, M. Khaldi, A. Azmani, Neural network-based decision support system for pre-diagnosis of psychiatric disorders // Proc. of Information Science and Technology (CIST), Third IEEE Intern. Colloquium, 20-22 Oct. 2014, pp. 102-106.

25 V. E. Ekong, U. G. Inyang, E. A. Onibere, Intelligent Decision Support System for Depression Diagnosis Based on Neuro-fuzzy-CBR Hybrid, Modern Applied Science, 6(7), 2012, pp. 79-88.

26 R. D. Ariyanti, S. Kusumadewi, I. V. Paputungan, Beck Depression Inventory Test Assessment Using Fuzzy Inference System, Proc. of Int. Conf. on Intelligent Systems Modeling and Simulation (ISMS), IEEE Computer Society, 2010, pp. 6-9.

27 B. A. Kobrinskii, A retrospective analysis of medical expert systems, News of artificial intelligence, 2, 2005, pp. 6-17.

28 L. V. Kan, Y. M. Kuznetcova, N. V. Chudova, Expert systems in the field of psycho-diagnostics, Artificial intelligence and decision-making, 2, 2010, pp. 26-35.

29 J. Thongkam, G. Xu, Y. Zhang, F. Huang, Toward breast cancer survivability prediction models through improving training space, Expert Syst. Appl., 36(10), 2009, pp. 12200-12209.

30 L. Parthiban, R. Subramanian, Intelligent heart disease prediction system using canfis and genetic algorithm, International Journal of Medical, Health, Biomedical and Pharmaceutical Engineering, 3, 2008, pp. 157160 .

31 K. Deb, A. Anand, D. Joshi, Computationally efficient evolutionary algorithm for real-parameter optimization, Evolutionary Computation, 4(10), 2002, pp. 371-395.

32 A. E. Yankovskaya, Test methods in pattern recognition in medical expert systems to cognitive graphics elements, Computer, chronicle, 8-9, 1994, pp. 61-83. (in Russian).
33 A. E. Yankovskaya, A. N. Kornetov, N. N. Il'inskikh, A.V. Silaeva, S. V. Kitler, S. S. Rakitin, On a question of the intellectual system construction for biopsychosocial express-diagnostics and depression prevention, Proceedings of Medical Cybernetics and interdisciplinary training of specialists for medicine: scientific conference proc. dedicated 25th anniversary of department of medical and biological Cybernetics (Nov. 14 - 15, Tomsk, Russia, 2013), 2013, pp. 39-45.

34 A. E. Yankovskaya, S. V. Kitler, A. A. Izyumov, N. M. Krivdyuk, Bases of construction intelligent medical systems based on soft computing and threshold logic, Proceedings of the Congress on Information Systems and Intelligent Technologies "IS\&IT'13" Issue 1, 2013, pp. 162-169.

35 I. A. Hodashinsky, P. A. Dudin, D. S. Sinkov, A. A. Zajcev and I. V. Gorbunov, "Construction fuzzy systems for predicting the effectiveness of non-drug Treatment," Computer Science and Control Systems. 3(33), 2012, pp. 140-150 (In Russian).

36 I. Hodashinsky, A. Anfilofyev, R. Meshcheryakov, I. Gorbunov, Designing Fuzzy Systems to Predict Efficiency of the NonPharmacological Treatment, Advanced Materials Research, 1085, 2015, pp. $465-470$.

37 A. Lemos, W. Caminhas, F. Gomide, Adaptive fault detection and diagnosis using an evolving fuzzy classifier, Information Sciences, 220, 2013, pp. 64-85.

38 L. Wang, L. Wu, D. Fu, Adaptive Learning by using a New Evolving Clustering Method, Journal of Computational Information Systems, Vol. 10 (21), 2014, pp. 9461-9468.

39 L. Zhu, L. Cao, J. Yang, J. Lei, Evolving soft subspace clustering, Applied Soft Computing, 14, Part B, 2014, pp. 210-228.

40 P. C. Young, Recursive Estimation and Time-Series Analysis: An Introduction for the Student and Practitioner. Second edition. New York: Springer-Verlag, 2011, 504 p.

41 P. Angelov, A. Ledezma, A. Sanchis, Creating evolving user behavior profiles automatically, IEEE Transactions on Knowledge and Data Engineering, 24(5), 2012, pp. 854-867.

42 N.K. Kasabov, Evolving Connectionist Systems for Adaptive Learning and Knowledge Discovery: Trends and Directions, Knowledge-Based Systems, 80, 2015, pp. 24-33.

43 A. Yankovskaya, A. Yamshanov, Family of 2-simplex cognitive tools and their application for decision-making and its justifications, Computer Science \& Information Technology (CS \& IT), Volume 6, Issue 1, Jan 02-03, 2016, Zurich, Switzerland, pp. 63-76.

44 S. V. Kondratenko, A. E. Yankovskaya, System of visualization TRIANG for decision-making substantiation with use cognitive graphics, Theses of the Third Conference on Artificial Intelligence, vol. 1, Tver, pp. 152-155 1992 (in Russian).

45 A. E. Yankovskaya, Transformation of features space in patterns space on the base of the logical-combinatorial methods and properties of some geometric figures, Proceedings of the International Conference Pattern Recognition and Image Analysis: New Information, Abstracts of the I All-Union Conference, Part II, pp. 178-181, Minsk, 1991, (in Russian).

46 A. E. Yankovskaya, M. E. Semenov, Decision-Making in Intelligent Training-Testing Systems based on Mixed Diagnostic Tests, Scientific and Technical Information Processing 40(6), 2013, pp. 329-336.

47 A. E. Yankovskaya, A. V. Yamshanov, N. M. Krivdyuk, Application of Cognitive Graphics Tools in Intelligent Systems, IJEIT, 3(7), January 2014, pp. 58-65.

48 A. E. Yankovskaya, "Logical tests and means of cognitive graphics in intelligence system," in Proc. 3rd All-Russian Conf. with international Participation "New Information Technologies in Discrete Structures Research" (Siberian Branch RAS, Tomsk, 2000), pp. 163-168.

49 A. E. Yankovskaya, "Logic-combinational probabilistic recognition algorithms," Pattern Recogn. Image Anal. 11 (1), 2001, pp. 123-126.

50 A. E. Yankovskaya, R. V. Ametov, Construction of Fault Tolerant Signal Feature Subsets, Pattern recognition and image analysis, 25(1), 2015, pp. $111-116$

51 I. A. Hodashinsky, R. V. Meshcheryakov, I. V. Gorbunov, Designing fuzzy rule-based classifiers using a bee colony algorithm", Informatics, Networking and Intelligent Computing proceedings of the 2014 international conference (INIC 2014), 16-17 November 2014, Shenzhen, China, CRC Press 2015, pp 25-34. 
52 P. Angelov, D. Filev, An Approach to Online Identification of TakagiSugeno Fuzzy Models, IEEE Transactions on Systems, Man, Cybernetic. B, 34(1), 2004, pp. 484-498.

53 D. Dovzan, I. Skrjanc, Recursive fuzzy c-means clustering for recursive fuzzy identification of time-varying processes, ISA Transactions, 50(2), 2011, pp. 159-169.

54 A. E. Yankovskaya, A. I. Gedike, R. V. Ametov, A. M. Bleikher, "IMSLOG-2002 Software Tool for Supporting Information Technologies of Test Pattern Recognition, Pattern Recognition and Image Analysis, 13(4), 2003, pp. 650-657.

55 A. E. Yankovskaya, D. V. Galkin, D. E. Chernogoryuk, Computer visualization and cognitive graphics tools for applied intelligent systems, Proceedings of the IASTED International Conferences on Automation, Control and Information Technology, 1, 2010, pp. 249-253. 